\title{
Influência do cetoprofeno na consolidação óssea de ulnas osteotomizadas de coelhos. Estudo qualitativo e quantitativo através de programas computacionais ${ }^{1}$
}

\author{
Luís Gustavo Rodrigues Pelissoni ${ }^{2}$ \\ Mário Jefferson Quirino Louzada ${ }^{3}$ \\ Adelina Maria da Silva \\ Luis Eduardo Barreto Martins ${ }^{5}$ \\ William Dias Belangero ${ }^{6}$
}

\begin{abstract}
Pelissoni LGR, Louzada MJQ, Silva AM, Martins LEB, Belangero WD. Influência do cetoprofeno na consolidação óssea de ulnas osteotomizadas de coelhos: estudo qualitativo e quantitativo através de programas computacionais. Acta Cir Bras [serial online] 2003 Set-Out;18(5). Disponível em URL: http://www.scielo.br/acb.
\end{abstract}

RESUMO - Objetivo: Acompanhar o processo de consolidação óssea de ulnas osteotomizadas de coelhos tratados com cetoprofeno, por meio de programas computacionais. Métodos: Os coelhos foram submetidos a osteotomia da ulna e divididos em dois grupos. No grupo A $(n=10)$ os animais foram tratados com cetoprofeno $\left(2 \mathrm{mg} . \mathrm{kg}^{-1}\right)$ durante cinco dias. O grupo $\mathrm{B}(\mathrm{n}=5)$ serviu como controle. Os animais foram radiografados um dia após a operação e a cada 7 dias até perfazer 28 dias. No ato da tomada radiográfica, junto à região a ser estudada, foram colocadas uma escada e uma cunha de alumínio que serviram como referencial de densidade óptica. As imagens radiográficas foram digitalizadas através de um "scanner". O programa ODR forneceu imagens tridimensionais e coloridas. Outro programa, CROMOX, avaliou a densidade mineral óssea da região da ostetotomia. Resultados: As imagens fornecidas pelo ODR proporcionaram melhor visualização da lesão e do processo de consolidação óssea. O programa CROMOX forneceu uma análise quantitativa ao calcular a densidade mineral do calo ósseo formado ao longo dos 28 dias de observação. Não houve diferença estatisticamente significante entre os valores de densidade mineral óssea das ulnas osteotomizadas dos coelhos tratados com cetoprofeno e do grupo controle, nas radiografias realizadas ao longo de 28 dias de pós-operatório. Conclusão: O cetoprofeno não interferiu no processo de formação do calo ósseo de ulnas osteotomizadas de coelhos.

DESCRITORES - Cetoprofeno. Osteototomia. Consolidação da fratura. Radiografia. Animal.

\section{Introdução}

A utilização de analgésicos como componentes da anestesia para a realização de procedimentos operatórios e o emprego dessas mesmas drogas para o tratamento da dor pós-operatória tem sido fortemente recomendado. A utilização de opióides e antiinflamatórios não esteróides têm aumentado nos últimos anos, principalmente em ortopedia, cujos procedimentos operatórios causam dor de elevada intensidade ${ }^{1,2,3}$.

Em casos de traumas ortopédicos acidentais ou provocados é rotina se verificar o estágio inicial da fratura e se acompanhar o processo de consolidação óssea por meio de análises radiográficas, que normalmente são qualitativas e dependem muito da experiência do profissional médico. Há mais de uma década nota-se um empenho no desenvolvimento de métodos quantitativos para avaliar o processo de consolidação da fratura ${ }^{4,5,6}$.

Unindo essas duas questões este estudo teve como objetivo acompanhar o processo de consolidação óssea em ulnas osteotomizadas de coelhos tratados com cetoprofeno, um antiinflamatório não esteróide de alto poder analgésico. O processo de consolidação óssea foi avaliado qualitativa e quantitativamente através de programas computacionais de processamento de 
imagens e análises numéricas, especialmente desenvolvidos.

\section{Métodos}

Foram utilizados 15 coelhos (Oryctolagus cuniculus) da linhagem Nova Zelândia, adultos, machos com peso médio de 4 quilos e idade média de 5 meses. A amostra foi distribuída aleatoriamente em dois grupos denominados grupo A $(n=10)$ e grupo $B(n=5)$. No grupo $\mathrm{A}$ os coelhos foram submetidos à osteotomia da ulna e tratados com cetoprofeno $\left(2 \mathrm{mg} \cdot \mathrm{kg}^{-1}\right.$, por via subcutânea). No grupo B (controle) os coelhos foram submetidos a osteotomia da ulna e receberam injeção subcutânea de solução fisiológica. Esses tratamentos foram realizados logo após o procedimento operatório e por mais cinco dias. Ambos os grupos foram tratados com o antibiótico enrofloxacina $\left(5 \mathrm{mg} \cdot \mathrm{kg}^{-1}\right.$, por via subcutânea) no pós-operatório imediato e durante três dias. Os animais foram acompanhados por um período de 4 semanas (28 dias).

Os coelhos foram anestesiados com quetamina na dose de $50 \mathrm{mg} . \mathrm{kg}^{-1}$, associada à xilazina, na dose de $10 \mathrm{mg} . \mathrm{kg}^{-1}$, por via intramuscular. Após preparação para técnica asséptica, a operação foi iniciada com uma incisão de pele longitudinal de $2 \mathrm{~cm}$ na região do terço médio da ulna. Após divulsão dos tecidos moles e exposição do osso, a osteotomia da ulna foi feita com um disco diamantado acoplado a uma caneta elétrica de baixa rotação de uso odontológico. Durante o procedimento, solução fisiológica foi gotejada no local para evitar necrose óssea por aquecimento. A tela subcutânea e a pele foram suturadas com fio de poliamida monofilamentar de $0,30 \mathrm{~mm}$ de diâmetro. Após o período de estudo, os animais foram submetidos a eutanásia pela administração intravenosa de superdose de tiopental sódico.

Os animais foram radiografados um dia após a operação e a cada 7 dias até perfazer 28 dias. No ato da tomada radiográfica, junto à região a ser estudada, foram colocadas, umas cunhas e umas escadas, ambas de alumínio, que serviram como referencial de densidade óptica. As imagens radiográficas foram digitalizadas através de um "scanner" HP acoplado a um adaptador para transparências da mesma marca. As imagens digitalizadas foram armazenadas com extensão TIFF.

O programa nomeado "ODR", em desenvolvimento, foi escrito no programa de análises numéricas MATLAB. Este programa processou as imagens digitalizadas das radiografias, fornecendo novas imagens tridimensionais e coloridas (Figura 1). Nos eixos $\mathrm{x}$ e y dessas imagens tridimensionais estão a localização superficial dos pontos da imagem originalmente digitalizada e no eixo $\mathrm{z}$ estão os valores de densidade óptica, expressos em milímetros de alumínio.

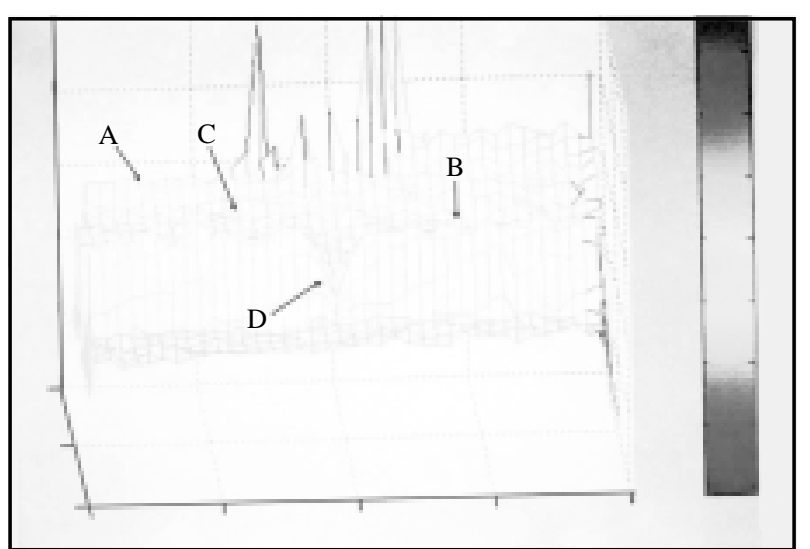

FIGURA 1 - Fotografia da tela do computador mostrando a imagem reconstruída pelo programa ODR da primeira tomada radiográfica, realizada 1 dia após a osteotomia, da ulna do coelho 1 do grupo B (controle). As letras A e B indicam a região do córtex ósseo, $\mathrm{C}$ indica a região do canal medular e D o local da osteotomia.

O programa nomeado "CROMOX”, especialmente desenvolvido, processou as imagens digitalizadas das radiografias para avaliar a densidade mineral óssea na região da osteotomia da ulna dos coelhos. Com o auxílio do "mouse", foram selecionadas as regiões de interesse: o calo ósseo na região da osteotomia da ulna e a escada de alumínio de 12 degraus, que serviu como referencial densitométrico. A área delimitada pelo mouse foi lida pelo programa "CROMOX" pixel a pixel, o qual forneceu o valor relativo à densidade óptica como média destas leituras. Os valores de densidade óptica foram expressos em níveis de cinza (até 256) (Figura 2). Os valores densitométricos obtidos foram convertidos para valores em espessura pelo computador, utilizando-se a metodologia descrita por LOUZADA ${ }^{7}$.

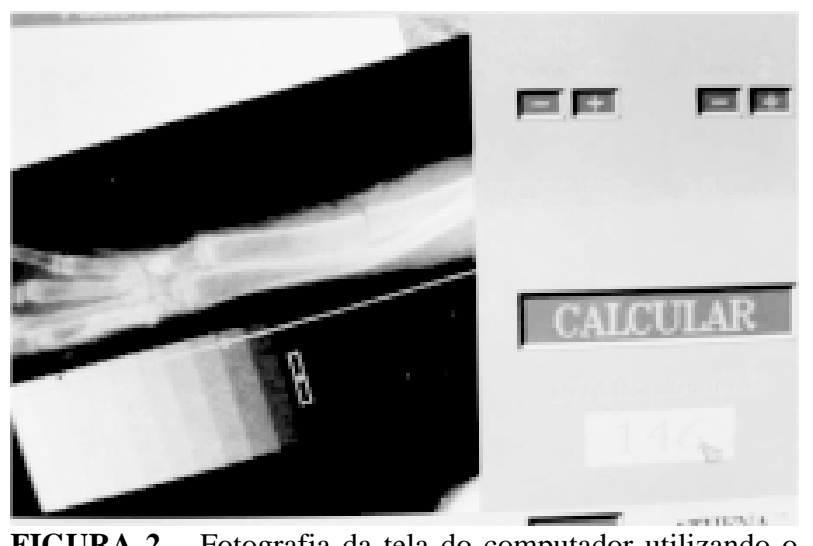

FIGURA 2 - Fotografia da tela do computador utilizando o programa CROMOX. A imagem mostra a radiografia da ulna operada e a ao lado da mesma, a escada de alumínio. Na região delimitada pelo "mouse" foi feita a leitura de densidade expressa em níveis de cinza. 
Para análise dos valores médios de densidade mineral óssea, em milímetros de alumínio, do calo ósseo das ulnas osteotomizadas foi aplicado o teste " $t$ "de Student, que comparou os dois grupos (cetoprofeno e controle) nos diferentes períodos de pós-operatório.
Fixou-se em 0,05 ou 5\% o nível de rejeição da hipótese de nulidade assinalando-se com um asterisco os valores significantes.

\section{Resultados}

TABELA 1 - Valores médios de densidade mineral óssea, em milímetros de alumínio, das ulnas dos coelhos do grupo A (tratados com cetoprofeno), medidos nas radiografias realizadas após períodos de pós-operatório de 1, $7,14,21$ e 28 dias.

\begin{tabular}{cccccc}
\hline Coelhos & 1dia & $\mathbf{7}$ dias & $\mathbf{1 4}$ dias & 21 dias & 28 dias \\
\hline T1 & 3,02 & 3,52 & 3,94 & 4,88 & 3,91 \\
T2 & 3,43 & 4,54 & 5,31 & 5,3 & 4,61 \\
T3 & 3,13 & 3,52 & 4,28 & 4,02 & 2,3 \\
T4 & 3,24 & 3,64 & 3,96 & 4,68 & 5,34 \\
T5 & 3,19 & 3,79 & 4,78 & 4,01 & 4,54 \\
T6 & 3,18 & 4,02 & 5,04 & 4,02 & 5,16 \\
T7 & 3,08 & 3,52 & 4,49 & 5,31 & 6,13 \\
T8 & 3,14 & 3,38 & 3,91 & 2,77 & 4,46 \\
T9 & 3,55 & 3,39 & 4,14 & 4,6 & $*$ \\
T10 & 3,05 & 3,7 & 4,54 & 4,73 & 4,15 \\
\hline
\end{tabular}

(*) perda de dado por morte do animal.

TABELA 2 - Valores médios de densidade mineral óssea, em milímetros de alumínio, das ulnas dos coelhos do grupo B (controle), medidos nas radiografias realizadas após períodos de pós-operatório de 1, 7, 14, 21 e 28 dias.

\begin{tabular}{cccccc}
\hline Coelhos & 1dia & 7 dias & 14 dias & 21 dias & 28 dias \\
\hline C1 & 3,64 & 3,34 & 5,74 & 6,04 & 5,45 \\
C2 & 2,82 & 4,14 & 4,98 & 5,14 & 5 \\
C3 & 2,96 & 3,64 & 4,11 & 4,73 & $*$ \\
C4 & 3,08 & 4,02 & 4,69 & 4,92 & 5,27 \\
C5 & 3,48 & 3,61 & 4,41 & 4,34 & 3,77 \\
\hline
\end{tabular}

(*) perda de dado por morte do animal.

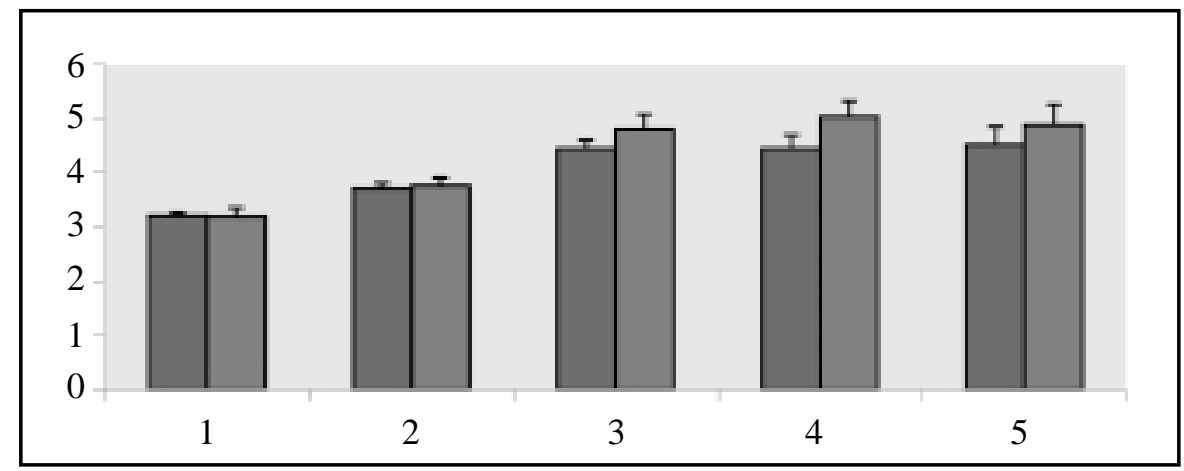

FIGURA 3 - Comparação entre as médias e erros padrão dos valores médios de densidade mineral óssea, em milímetros de alumínio, das ulnas dos coelhos dos grupos A (tratados com cetoprofeno) e B (controle), medidos nas tomadas radiográficas realizadas no pós-operatório: 1 (1 dia), 2 (7 dias), 3 (14 dias), 4 (21 dias) e 5 (28 dias). 


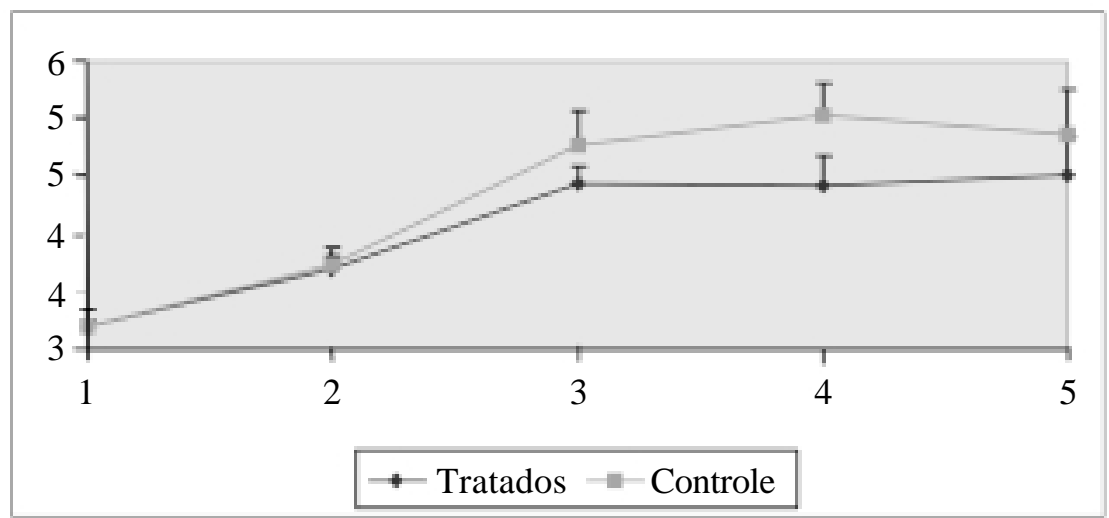

FIGURA 4 - Evolução dos valores médios de densidade mineral óssea, em milímetros de alumínio, das ulnas dos coelhos dos grupos A (tratados com cetoprofeno) e B (controle), medidos nas tomadas radiográficas realizadas no pós-operatório: 1 (1 dia), 2 (7 dias), 3 (14 dias), 4 (21 dias) e 5 (28 dias).

TABELA 3 - Médias e desvios padrão dos valores médios de densidade mineral óssea, em milímetros de alumínio, das ulnas dos coelhos dos grupos A (tratados com cetoprofeno) e B (controle), medidos nas radiografias realizadas após períodos de pós-operatório de 1, 7, 14, 21 e 28 dias.

\begin{tabular}{cllc}
\hline $\begin{array}{c}\text { Tomadas radiográficas } \\
\text { no pós-operatório }\end{array}$ & Tratado & Controle & P \\
\hline 1 dia & $3,20+/-0,05$ & $3,20+/-0,16$ & 0,97 \\
7 dias & $3,70+/-0,11$ & $3,75+/-0,15$ & 0,803 \\
14 dias & $4,44+/-0,15$ & $4,79+/-0,28$ & 0,255 \\
21 dias & $4,43+/-0,25$ & $5,03+/-0,28$ & 0,152 \\
28 dias & $4,51+/-0,34$ & $4,87+/-0,38$ & 0,557 \\
\hline
\end{tabular}

Teste $\mathrm{t}$ de Student $(\mathrm{p}<0,05)$

\section{Discussão}

O programa ODR transformou as imagens digitalizadas das radiografias em novas imagens tridimensionais e coloridas. Nestas imagens, o local da osteotomia ficava bem visível e foi possível acompanhar claramente a formação do calo ósseo. A apresentação dos valores de densidade óptica em cores possibilitou uma melhor visualização e avaliação qualitativa dos níveis de densidade mineral óssea. Nas imagens coloridas foram realizadas comparações qualitativas entre a região osteotomizada e as regiões adjacentes preservadas, que serviram como referencial visual do estado evolutivo do processo de consolidação óssea.

A avaliação quantitativa do processo de consolidação óssea realizada pelo programa CROMOX mostrouse rápida, simples e barata. Neste programa as imagens radiográficas digitalizadas foram analisadas a partir dos níveis de cinza observados em uma determinada área.
Os valores obtidos nessa área foram confrontados com aqueles fornecidos pela escada de alumínio, que serviu como referencial densitométrico. Finalmente, estes valores de densidade óptica foram convertidos em valores de espessura, expressos em milímetros de alumínio.

A tomografia computadorizada também pode ser utilizada para avaliar quantitativamente a consolidação óssea, conforme estudo realizado por $\mathrm{AUGAT}^{4} \mathrm{em}$ tíbias osteotomizadas de ovinos. BOHM $^{5}$ conseguiu calcular a resistência ao encurvamento de ossos fraturados em processo de consolidação óssea, a partir dos dados obtidos pela tomografia computadorizada.

Outro método para avaliar quantitativamente a consolidação óssea foi apresentado no estudo de den BOER $^{6}$, que utilizaram a taxa de absorção de raios $\mathrm{X}$ de dupla energia. Contudo, estas duas técnicas exigem equipamentos mais caros, o que restringe a sua utilização.

Os medicamentos antiinflamatórios não esteróides (AINES) inibem tanto a atividade da ciclooxigenase-1 
(COX-1; constitucional) quanto da ciclooxigenase-2 (induzida em presença da inflamação) e desta forma as sínteses das prostaglandinas e do tromboxano. A inibição da COX-2 parece mediar as ações antipirética, analgésica e antiinflamatória dos AINES, porém a inibição simultânea da COX-1 provoca efeitos colaterais indesejáveis, principalmente ulceração e intolerância gastrintestinais. Embora também seja um inibidor da ciclooxigenase, o cetoprofeno parece estabilizar as membranas lipossômicas e pode antagonizar as ações da bradicinina ${ }^{8}$.

Os efeitos analgésicos do cetoprofeno já foram testados em vários experimentos ${ }^{1,2,3}$ contudo, estudos sobre o efeito deste antiinflamatório não esteróide na consolidação da fratura não foram encontrados na revisão bibliográfica efetuada.

Constatou-se no presente experimento que os valores médios da densidade mineral óssea, em milímetros de alumínio, aumentaram ao longo das semanas de estudo, tanto nos coelhos tratados com cetoprofeno como naqueles do grupo controle. Quando as médias dos valores de densidade mineral óssea dos dois grupos foram comparadas, verificou-se que não houve diferença estatisticamente significante em nenhum dos períodos de pós-operatório.

Este resultado difere do observado com o uso sistêmico prolongado de corticosteróides. WATERS ${ }^{9}$ constatou o efeito adverso dos corticosteróides na consolidação óssea de ulnas osteotomizadas de coelhos, através de estudos radiográficos e biomecânicos.

A patofisiologia da osteoporose induzida por corticosteróides já foi bem estudada e provavelmente mecanismos similares devem provocar efeitos adversos dos corticosteróides na consolidação da fratura. Os corticosteróides inibem diretamente a atividade dos osteoblastos, diminuindo a formação óssea. Além disso, devido à sua inibição da absorção do cálcio no intestino, os corticosteróides aumentam a secreção de hormônio paratireoidiano, que por sua vez atua sobre os osteoclastos para aumentar a reabsorção óssea ${ }^{9,10}$.

A ação antiinflamatória dos corticosteróides se deve não apenas a liberação diminuída de prostaglandinas mas também a inibição da produção de citocinas, reagentes de fase aguda, histamina, leucotrienos, molécula 1 de adesão leucocitária endotelial e molécula 1 de adesão intracelular. Os corticosteróides também suprimem a síntese do DNA induzida pelo fator de crescimento e a proliferação de fibroblastos ${ }^{9,10}$.

Este trabalho vem atestar a eficácia da utilização de programas computacionais para as análises qualitativas e quantitativas do processo de consolidação da fratura. As imagens fornecidas pelo ODR proporcionaram melhor visualização da lesão e de todo o processo de consolidação óssea. A análise quantitativa permitiu calcular a densidade mineral do calo ósseo formado de uma maneira simples e de custo reduzido. Através dessa análise quantitativa verificou-se que o cetoprofeno não interferiu no processo de formação do calo ósseo.

\section{Conclusão}

O cetoprofeno não interferiu no processo de formação do calo ósseo de ulnas osteotomizadas de coelhos.

\section{Referências}

1. Niemi L, Tuominen M, Pitkanen M, Rosenberg PH. Comparison of parenteral diclofenac and ketoprofen for postoperative pain relief after maxillofacial surgery. Acta Anaest Scand 1995; 39:96-9.

2. Matsuda EI, Fantoni DT, Futema F, Migliati ER, Ambrósio A, Almeida TI. Estudo comparativo entre o ketoprofeno e o flunixin meglumine no tratamento da dor pós-operatória de cães submetidos a cirurgia ortopédica. Clin Vet 1999; 4:19-22.

3. Alves AS, Campello, RAV, Mazzanti A, Alievi MM, Faria RX, Stedille R, Braga FA. Emprego do antiinflamatório não esteróide ketoprofeno na analgesia preventiva em cães. Ciência Rural 2001; 31:439-44.

4. Augat P, Merk J, Genant HK, Claes L. Quantitative assessment of experimental fracture repair by peripheral computed tomograghy. Calcif Tissue Int 1997; 60:194-9.

5. Bohm AM, Jungkunz B. Bending stiffness of healing fractures can be calculated from quantitative computed tomography. Eur J Radiol 1999; 30:28-32

6. den Boer FC, Patka P, Bakker FC, Wippermann BW, van Lingen A, Vink GQM, Boshuizen K, Haarman HJTHM. New segmental long bone defect model in sheep: quantitative analysis of healing with dual energy X-ray absorptiometry. J Orthop Res 1999; 17:654-60.

7. Louzada MJQ, Pelá CA, Belangero WD, Santos-Pinto R., Metodologia para avaliação de densidade em imagens radiográficas. Rev Bras Engenharia/Caderno Engenharia Biomédica 1998; 14:37-47.

8. Insel PA. Fármacos analgésicos, antipiréticos e antiinflamatórios e medicamentos usados no tratamento da gota. In: Hardman JG, Limbird LE, Molinoff PB, Ruddon RW. As bases farmacológicas da terapêutica. 9ed. Mexico: McGrawHill; 1996. p 450-80.

9. Waters RV, Gamradt SC, Asnis P, Vickery BH, Avnur Z, Hill E Bostrom M. Systemic corticosteroids inhibit bone healing in a rabbit ulnar osteotomy model. Acta Orthop Scand 2000; 71:317-21

10. Schimmer BP, Parker K. Hormônio adrenocorticotrófico; esteróides adrenocorticais e seus análogos sintéticos; inibidores da síntese e das ações dos hormônios adrenocorticais. In.: Hardman JG, Limbird LE, Molinoff PB, Ruddon RW. As bases farmacológicas da terapêutica. 9ed. Mexico: McGraw-Hill; 1996. p 1082-102

\section{Agradecimento}

A Sílvia Helena Venturoli Perri, Professora Doutora da Disciplina de Bioestatística da UNESP-Campus de Araçatuba, pela realização da análise estatística do presente trabalho. 
Pelissoni LGR, Louzada MJQ, Silva AM, Martins LEB, Belangero WD. Influence of ketoprofen on ulnar osteotomy healing in rabbits: qualitative and quantitative study using image analysis softwares. Acta Cir Bras [serial online] 2003 Sept-Oct;18(5). Available from URL: http://www.scielo.br/acb.

ABSTRACT - Purpose: Assessment of ulnar osteotomy healing in rabbits treated with ketoprofen, by image analysis softwares. Methods: The rabbits were submitted to ulnar osteotomy and divided in 2 groups. Group A $(\mathrm{n}=10)$ was treated with ketoprofen $\left(2 \mathrm{mg} \cdot \mathrm{kg}^{-1}\right)$ during 5 days. Group B $(\mathrm{n}=5)$ was the control. Radiographs were taken one day after the surgery and then every each 7 days until a total of 28 days. An aluminum step wedge and an aluminum wedge were placed at the osteotomy site to serve as optical densitometry references. The radiographic images were scanned and processed with ODR software in order to obtain tridimensional and color images. Next, the average radiographic density in the area of the osteotomy was quantified using CROMOX software. Results: The radiographic images processed through ODR software resulted in a better visualization of the lesion and bone healing. Additionaly, CROMOX software allowed quantitative analysis of the osseous callus density along the 28 days of observation. We have observed no statistical significant diference between the values of osseous density of the ulnar osteotomy from ketoprofen-treated rabbits and the controls, in the radiographs taken during the 28 days of postoperative. Conclusion: Taken together our results have shown that treatment with ketoprofen does not impair ulnar osteotomy healing in rabbits.

KEY WORDS - Ketoprofen. Osteotomy. Fracture healing. Radiography. Animal.

Conflito de interesse: nenhum

Correspondência:

Fonte de financiamento: nenhuma

Mário Jefferson Quirino Louzada

Rua Clóvis Pestana, 793

16050-680 Araçatuba - SP

louzada@fmva.unesp.com

Data do recebimento: 20/07/2003

Data da revisão: 01/08/2003

Data da aprovação: 12/08/2003 